\title{
Nanoscale space charge generation in local oxidation nanolithography
}

\author{
Marco Chiesa and Ricardo Garcia ${ }^{\text {a) }}$ \\ Instituto de Microelectrónica de Madrid, CSIC, Isaac Newton, 8 Tres Cantos, Madrid 28760, Spain
}

(Received 6 May 2010; accepted 14 June 2010; published online 1 July 2010)

\begin{abstract}
We have measured the surface potential and the space charge generated during the first stages of atomic force microscopy field-induced oxidation. Space charge densities are about $10^{17} \mathrm{~cm}^{-3}$ for oxidation times below $10 \mathrm{~ms}$. In a dry atmosphere, the surface potential is negative. However, in humid air the surface potential could be either positive or negative. This effect is attributed to a screening effect of the water molecules. These results explain and support the use of local oxidation patterns as templates for building molecular architectures. They also establish the space charge build up as an intrinsic feature in local oxidation experiments. () 2010 American Institute of Physics. [doi:10.1063/1.3459976]
\end{abstract}

Atomic force microscopy (AFM) oxidation nanolithography is a robust and flexible tip-based nanofabrication method $^{1-5}$ that is used to fabricate high resolution patterns and nanoscale devices. Thus, templates to build molecular architectures ${ }^{6,7}$ resist masks, ${ }^{8}$ nanomechanical resonators, ${ }^{9,10}$ or several nanoelectronic devices and transistors ${ }^{11-14}$ have been fabricated by local oxidation. In AFM oxidation, the tip is used as a cathode and the water meniscus formed between the tip and the surface is the source of the oxyanions species. ${ }^{15}$ The strong localization of the electrical field lines near the tip apex and the lateral confinement of the oxyanions species within the liquid meniscus give rise to a nanometer-size oxide dot. ${ }^{16-20}$ The kinetics of the oxidation process is influenced by the generation of a space charge. ${ }^{21-23}$ The model by Dagata et al. ${ }^{21,24}$ considers two competing mechanisms, a fast oxidation process that applies to the initial stages (below $1 \mathrm{~s}$ ) and a slower, indirect process that applies for longer oxidation times and involves space charge. Dubois and Bubendorff's model is based on a charge trapping-detrapping mechanism. ${ }^{23}$ Most of the AFM oxidation nanolithography applications involve oxidation times below $1 \mathrm{~s}$. Recently, local oxide patterns have been used as high resolution templates for the growth of functional materials such as protein carriers ${ }^{7}$ or single molecule magnets. ${ }^{25}$ The underlying mechanism behind the organization of molecular architectures is based on the electrostatic interactions between the charged or polarized molecules and the space charges trapped inside the local oxides. However, there is no experimental evidence that supports the existence of space charges for short oxidation times. In addition, the sign of the charge has not been measured.

Here, we perform Kelvin probe force microscopy (KPFM) experiments to determine the space charge sign and density. The measurements establish the presence of a space charge build up during the earlier stages of the oxidation process (below $10 \mathrm{~ms}$ ). The sign of the apparent space charge depends on the relative humidity inside the chamber where the KPFM measurements are performed. In dry conditions $\left(\mathrm{N}_{2}\right.$ gas environment) the observed charge is always negative. However, in the presence of water vapor, both positive and negative charges are measured. This variability is attributed to the screening effect of the water layer adsorbed on

${ }^{a)}$ Electronic mail: ricardo.garcia@imm.cnm.csic.es. the silicon oxide pattern and the silicon surfaces. The present findings, on one hand, explain and support the use of local oxides as templates for molecular architectures. On the other hand, they establish the existence of a significant space charge build up $\left(\sim 10^{17} \mathrm{~cm}^{-3}\right)$ during the first stages of the local oxidation process.

The experiments were performed with a dynamic atomic force microscope operated in the low amplitude solution (noncontact) and with additional circuits to perform the oxidation. ${ }^{26}$ The microscope was placed into a closed box with inlets for dry and water vapor-saturated nitrogen. To fabricate the oxidation patterns, the relative humidity in the AFM chamber was kept above 50\%. Noncontact AFM oxidations were performed with doped $n^{+}$-type silicon cantilevers (Nanosensors, Germany.) The force constant $k$ and the resonant frequency $f_{0}$ were about $30 \mathrm{~N} / \mathrm{m}$ and $300 \mathrm{kHz}$, respectively. The cantilever was excited at its resonant frequency. The silicon (100) samples were of $n$-type and $p$-type with a resistivity, respectively, of $1-10 \Omega \mathrm{cm}$ and $0.1-1 \Omega \mathrm{cm}$. The patterns were generated by applying a sequence of voltage $\mathrm{V}$ pulses (tip negative). For the $n$-type we applied a sequence of voltage pulses of $27.0 \mathrm{~V}$ for $1.5 \mathrm{~ms}$ and for the p-type $19.5 \mathrm{~V}$ and $5 \mathrm{~ms}$. Between the pulses, the tip was displaced laterally by about $8 \mathrm{~nm}$. To perform the KPFM measurements in a dry atmosphere, the chamber was first purged from water vapor by flushing dry $\mathrm{N}_{2}$ for about 60 $\min$. This reduced the relative humidity $(\mathrm{RH})$ below $1 \%$.

Figure 1 shows a scheme of the AFM oxidation process and the KPFM measurements. AFM oxidation experiments were performed at a $\mathrm{RH}$ above $50 \%$ while KPFM measurements were performed in either a dry $\mathrm{N}_{2}$ environment or at
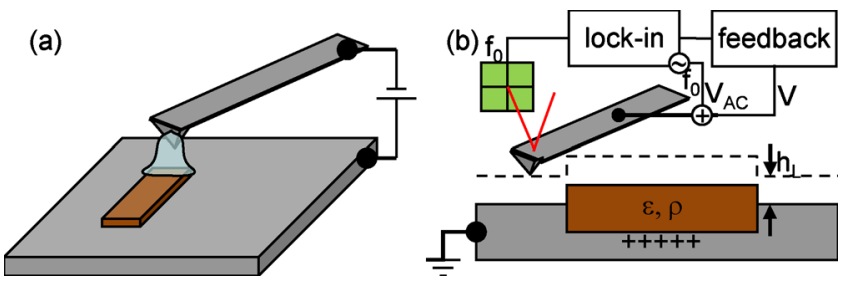

FIG. 1. (Color online) (a) Scheme of AFM oxidation nanolithography. The formation of a water meniscus between the tip and the surface and the anodic oxidation is driven by the application of an external voltage. (b) Scheme of KPFM to measure the sign and the charge density of the local oxides. 
$\mathrm{RH}$ of $35 \%$ to $45 \%$. KPFM experiments were performed in double pass with a lift height $h_{L}$ of $20 \mathrm{~nm}$.

KPFM experiments provide a direct measurement of the differences in the work function across a heterogeneous surface $^{27,28}$ and of the surface potential in presence of trapped charge. ${ }^{29}$ A relationship between the surface potential and the space charge within the oxide can be calculated by solving the Poisson equation,

$$
\frac{d E}{d z}=\frac{\rho}{\varepsilon \varepsilon_{0}},
$$

where $E$ is the electric field, $\rho$ the charge density, and $\varepsilon$ and $\varepsilon_{0}$ are, respectively, the relative dielectric constant of the silicon oxide and the dielectric constant of the vacuum. By integrating Eq. (1) with the boundary condition that in the region comprised between the oxide surface and the KPFM tip there is no field $[E(d)=0],{ }^{29}$ it is deduced

$$
E(z)=-\frac{d V}{d z}=\frac{\rho}{\varepsilon \varepsilon_{0}} z-\frac{\rho}{\varepsilon \varepsilon_{0}} d .
$$

Then, by assuming a uniform charge density, the following relationship between the surface potential $V$ and the space charge is deduced:

$$
\rho=\frac{2 \varepsilon \varepsilon_{0}}{d^{2}} V,
$$

where $d$ is the total thickness of the oxide and $z$ is the spatial coordinate along the oxide thickness $(z=0$ at the silicon/ oxide interface). Thus, the signs of the space charge and the surface potential coincide (the silicon wafer is grounded).

Figure 2 shows the topography and the surface potential measurements of two rectangular patterns performed on a $n$-type [Figs. 2(a)-2(f)] and a $p$-type $\mathrm{Si}(100)$ substrates [Figs. $2(\mathrm{~g})-2(\mathrm{l})]$. The surface potential difference between the bare silicon and the oxide patterns depends on the presence of water vapor in the KPFM chamber. In dry nitrogen, the surface potential difference is about $20 \mathrm{mV}$ higher than in humid air. This suggests the presence of an electrostatic interaction between the water molecules adsorbed on the oxide pattern and the KPFM probe. ${ }^{30}$ In addition, in humid air we observe some variability in the sign of the surface potential. In approximately half of the samples, the effective surface potential was positive while in the other half the surface potential was negative (Fig. 3). However, in dry $\mathrm{N}_{2}$ the surface potential of the oxide is always more negative than that of the bare silicon, irrespective of both the sign measured in air and the type of the doping of the silicon. This clearly indicates that the oxide always contains a negative trapped charge. This negative charge is likely to be due to the oxyanions which have not reached the silicon/oxide interface. Furthermore, the preferential deposition of molecular materials such as ferritin at $\mathrm{pH}=3$ and polycationic $\mathrm{Mn}_{12}$ on AFM oxide patterns ${ }^{7,25}$ is consistent with an attractive electrostatic interaction between the positively charged molecules and the negatively charged local oxide patterns.

In experiments performed in air, we attribute the decrease in the surface potential and/or its change in sign with respect to dry environments to the screening effect of the adsorbed water on the silicon oxide pattern and on the silicon surface. This effect could be considered a measurement artifact. It does not alter the sign of the charge inside of the oxide which remains negative.
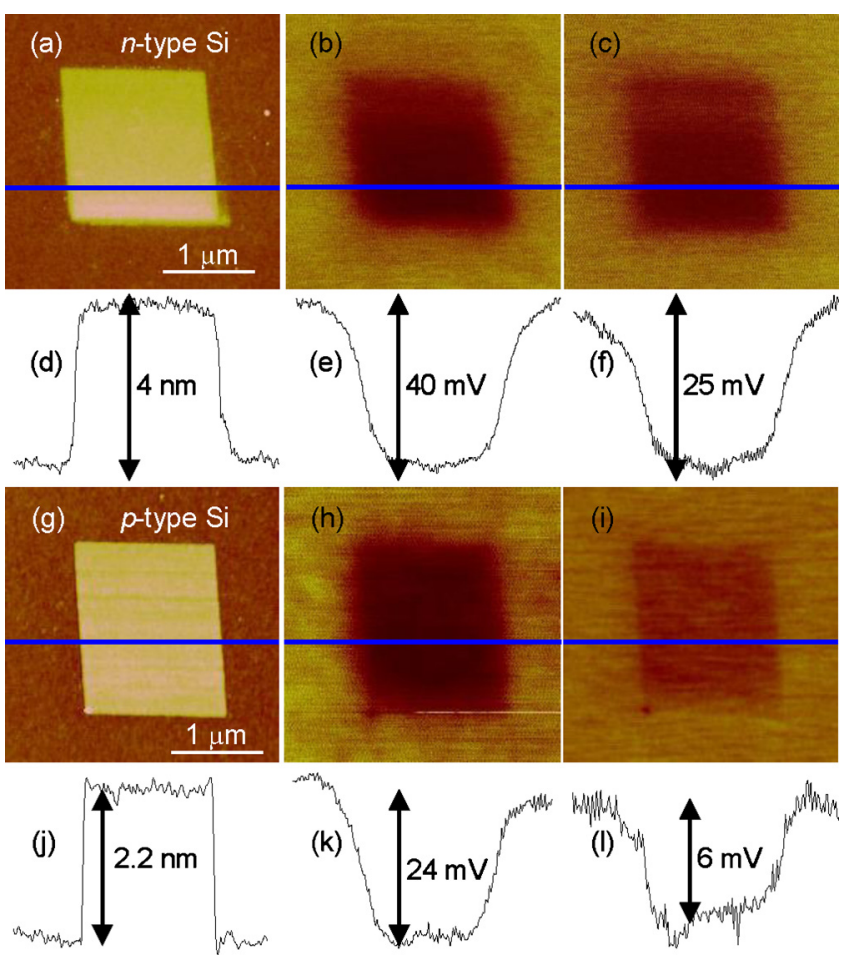

FIG. 2. (Color online) (a) AFM topographic image of a local oxide pattern fabricated on a $n$-type $\mathrm{Si}(100)$ substrate. (b) Surface potential image of the pattern shown in (a) obtained in a dry $\mathrm{N}_{2}$ (gas) environment. (c) Surface potential image of the pattern shown in (a) obtained in air at a $\mathrm{RH}$ of $37 \%$. [(d)-(f)] Profiles across the section drawn in (a)-(c), respectively. (g) AFM topographic image of a local oxide pattern fabricated on a $p$-type $\mathrm{Si}(100)$ substrate. (h) Surface potential image of the pattern shown in (g) obtained in a dry $\mathrm{N}_{2}$ environment. (i) Surface potential image of the pattern shown in (g) obtained in air at a RH of $44 \%$. [(j)-(1)] Profiles across the section drawn in (g)-(i), respectively.

The surface potential and the topographical data extracted from Fig. 2, together with Eq. (3), allow us to estimate the space charge density within the oxide. By considering that the topographical height of the oxide pattern represents $60 \%$ of its total thickness, ${ }^{3}$ and assuming $\varepsilon=3.9$ for an AFM oxide, a space charge density of $8 \times 10^{17} \mathrm{~cm}^{-3}$ is obtained for the $p$-type substrate while, for the $n$-type silicon, a density of $4 \times 10^{17} \mathrm{~cm}^{-3}$ is obtained.

In short, we have measured the space charge density during the first stages of local oxidation experiments. Two

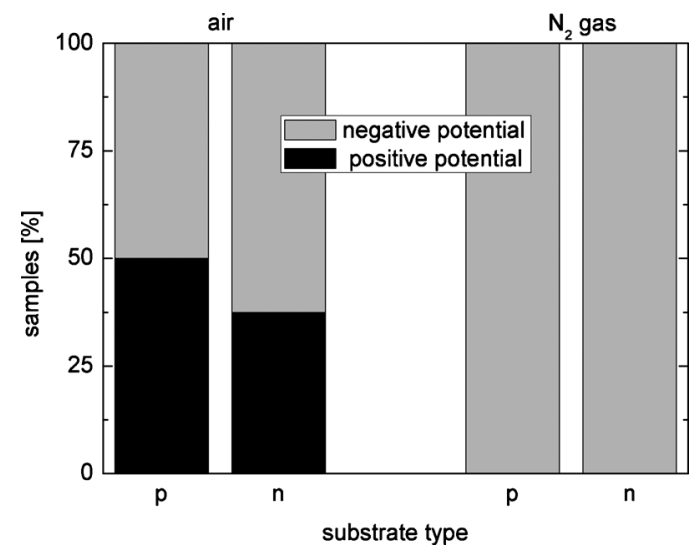

FIG. 3. Histogram of the sign of the surface potential difference between the local oxide patterns and the silicon as a function of the presence of water vapor in the KPFM chamber. 
major results have been found. First, the space charge density is of the order of $10^{17} \mathrm{~cm}^{-3}$ for voltage pulses in the ms range. Second, in the absence of water adsorbed on the oxide pattern, the effective surface potential is negative which implies a negative space charge. However, if the measurements are performed in air at a relative humidity above $10 \%$, the sign of the effective surface potential could be either positive or negative. This variability is attributed to a screening effect of the adsorbed water on the silicon oxide. These results explain and support the use of local oxidation patterns as templates for building molecular architectures. They also establish that the space charge build up is an intrinsic feature of local oxidation experiments.

We acknowledge financial support from the Ministerio de Ciencia, Investigación e Innovación (Grant Nos. MAT2009-08650 and CTQ2007-31076), the CSIC (Grant No. PIF08-008), and the Comunidad de Madrid (Grant No. S-2009-MAT-1467).

${ }^{1}$ F. C. Simeone, C. Albonetti, and M. Cavallini, J. Phys. Chem. C 113, 18987 (2009)

${ }^{2}$ A. Zeira, D. Chowdhury, R. Maoz, and J. Sagiv, ACS Nano 2, 2554 (2008).

${ }^{3}$ R. Garcia, R. V. Martinez, and J. Martinez, Chem. Soc. Rev. 35, 29 (2006).

${ }^{4}$ A. A. Tseng, A. Notargiacomo, and T. P. Chen, J. Vac. Sci. Technol. B 23, 877 (2005).

${ }^{5}$ D. Wouters and U. S. Schubert, Angew. Chem., Int. Ed. 43, 2480 (2004).

${ }^{6}$ S. Liu, R. Maoz, and J. Sagiv, Nano Lett. 4, 845 (2004).

${ }^{7}$ R. V. Martínez, J. Martínez, M. Chiesa, R. Garcia, E. Coronado, E. PinillaCienfuegos, and S. Tatay, Adv. Mater. 22, 588 (2010).

${ }^{8}$ I. Suez, M. Rolandi, S. A. Backer, A. Scholl, A. Doran, D. Okawa, A. Zettl, and J. M. J. Fréchet, Adv. Mater. 19, 3570 (2007).

${ }^{9}$ M. Villarroya, F. Pérez-Murano, C. Martín, Z. Davis, A. Boisen, J. Esteve, E. Figueras, J. Montserrat, and N. Barniol, Nanotechnology 15, 771
(2004).

${ }^{10}$ L. Pellegrino, M. Biasotti, E. Bellingeri, C. Bernini, A. S. Siri, and D. Marré, Adv. Mater. 21, 2377 (2009)

${ }^{11}$ L. Pellegrino, Y. Yanagisawa, M. Ishikawa, T. Matsumoto, H. Tanaka, and T. Kawai, Adv. Mater. 18, 3099 (2006).

${ }^{12}$ C. Delacour, J. Claudon, J.-P. Poizat, B. Pannetier, V. Bouchiat, R. Espiau de Lamaestre, J.-C. Villegier, M. Tarkhov, A. Korneev, B. Voronov, and G. Gol'tsman, Appl. Phys. Lett. 90, 191116 (2007).

${ }^{13}$ J. Martinez, R. V. Martínez, and R. Garcia, Nano Lett. 8, 3636 (2008).

${ }^{14}$ J. T. H. Tsai, B. H. B. Lee, and M. S. Yang, Phys. Rev. B 80, 245215 (2009).

${ }^{15}$ M. Calleja, M. Tello, and R. García, J. Appl. Phys. 92, 5539 (2002).

${ }^{16}$ J. A. Dagata, T. Inoue, J. Itoh, K. Matsumoto, and H. Yokoyama, J. Appl. Phys. 84, 6891 (1998).

${ }^{17}$ A. Garcia-Martin and R. Garcia, Appl. Phys. Lett. 88, 123115 (2006).

${ }^{18}$ T. Cramer, F. Zerbetto, and R. García, Langmuir 24, 6116 (2008).

${ }^{19}$ A. Orians, C. B. Clemons, D. Golovaty, and G. W. Young, Surf. Sci. 600, 3297 (2006)

${ }^{20}$ S. Lee, E. Pyo, J. O. Kim, J. Noh, H. Lee, and J. Ahn, J. Appl. Phys. 101, 044905 (2007).

${ }^{21}$ J. A. Dagata, F. Perez-Murano, G. Abadal, K. Morimoto, T. Inoue, J. Itoh, and H. Yokoyama, Appl. Phys. Lett. 76, 2710 (2000).

${ }^{22}$ C. R. Kinser, M. J. Schmitz, and M. C. Hersam, Adv. Mater. 18, 1377 (2006)

${ }^{23}$ E. Dubois and J.-L. Bubendorff, J. Appl. Phys. 87, 8148 (2000).

${ }^{24}$ J. A. Dagata, F. Perez-Murano, C. Martin, H. Kuramochi, and H. Yokoyama, J. Appl. Phys. 96, 2386 (2004).

${ }^{25}$ R. V. Martínez, F. García, R. García, E. Coronado, A. Forment-Aliaga, F. M. Romero, and S. Tatay, Adv. Mater. 19, 291 (2007).

${ }^{26}$ M. Tello, R. Garcia, J. A. Martín-Gago, N. F. Martínez, M. S. MartínGonzález, L. Aballe, A. Baranov, and L. Gregoratti, Adv. Mater. 17, 1480 (2005).

${ }^{27}$ M. Nonnenmacher, M. P. O'Boyle, and H. K. Wickramasinghe, Appl. Phys. Lett. 58, 2921 (1991).

${ }^{28}$ V. Palermo, M. Palma, and P. Samorì, Adv. Mater. 18, 145 (2006).

${ }^{29}$ L. Bürgi, T. Richards, M. Chiesa, R. H. Friend, and H. Sirringhaus, Synth. Met. 146, 297 (2004).

${ }^{30}$ H. Sugimura, Y. Ishida, K. Hayashi, O. Takai, and N. Nakagiri, Appl. Phys. Lett. 80, 1459 (2002). 